\title{
OS ASPECTOS MORFOSINTÁTICOS E LÉXICOS DO SPANGLISH NA AULA DE E/LE EM UM CONTEXTO UNIVERSITÁRIO
}

\author{
LOS ASPECTOS MORFOSINTÁCTICOS Y LÉXICOS DEL \\ SPANGLISH EN LA CLASE DE E/LE EN UN CONTEXTO \\ UNIVERSITARIO
}

\author{
THE MORPHOSYNCTACTIC AND LEXICAL ASPECTS OF \\ SPANGLISH IN A SPANISH AS A SECOND LANGUAGE LESSON IN A \\ UNIVERSITY SETTING
}

Cristian TUGUES RODRÍGUEZ ${ }^{1}$

RESUMO: O presente estudo concentra-se na justificativa de incluir nas aulas de segundas línguas/línguas estrangeiras as línguas em contato. Em concreto, mostramos a nossa própria experiência levando o Spanglish dos Estados Unidos à uma aula de espanhol como língua estrangeira $(E / L E)$ num contexto universitário. Para isso, oferecemos uma unidade didática onde o aluno é capaz de aprender sobre esta variedade popular de espanhol estado-unidense ao mesmo tempo em que lhe ensinamos os diferentes mecanismos e recursos linguísticos que o formam para assim classificar algumas amostras reais e literárias em arquivos lexicográficos. Como resultado pretendemos trazer, além das variedades linguísticas e pan-hispânicas para nossa classe de E/LE, a concepção dentro da sala de aula de que as linguagens são algo global e em contínua evolução.

PALAVRAS-CHAVE: Espanhol como língua estrangeira $(E / L E)$. Espanhol estadounidense. Línguas em contato. Spanglish. Variedades do espanhol. Dialetologia pedagógica.

RESUMEN: La presente investigación se centra en la justificación de llevar las lenguas en contacto a las aulas de segundas lenguas. En concreto mostramos nuestra propia experiencia llevando el Spanglish de los Estados Unidos a una clase de español como lengua extranjera (E/LE) en un contexto universitario. Para ello, ofrecemos una unidad didáctica donde el estudiante es capaz de aprender sobre esta variedad popular del español estadounidense al mismo tiempo que le enseñamos los diferentes mecanismos y recursos lingüísticos que lo forman para poder clasificar algunas muestras reales y literarias en fichas lexicográficas. Con ello, pretendemos acercar no solo las variedades lingüísticas y panhispánicas a nuestra clase de E/LE, sino la concepción en el aula de que las lenguas son algo global y en una continua evolución.

1. Universitat de València (UV), València - Comunitat Valenciana - Espanha. Doutorando do programa de Estudos Hispânicos Avançados. Universidade Estadual Paulista (Unesp), Araraquara - SP - Brasil. Leitor de espanhol AECID. ORCID: https://orcid.org/0000-0002-6710-8601. E-mail: cristian.tugues@gmail.com ; cristuro@alumni.uv.es 
PALABRAS CLAVE: Español como lengua extranjera (E/LE). Español estadounidense. Lenguas en contacto. Spanglish. Variedades del español. Dialectología pedagógica.

ABSTRACT: This research focuses on the justification of bringing the linguistic contact into second language classrooms. In particular, we show our own experience by taking Spanglish from the United States into a Spanish as a foreign language (E/LE) lesson within a university setting. Therefore, to carry out this, we offer a lesson planning where the student is able to learn about this so called popular American Spanish at the same time we teach the different mechanisms and linguistic resources that form it so as to classify some real and literary samples in lexicographic charts. Thus, we intend to bring not only the linguistic and panhispanic varieties to our E/LE lesson, but also the conception in the classroom that languages are something global in a continuous evolution.

KEYWORDS: Spanish as a foreign language (E/LE). North-American Spanish. Languages in contact. Spanglish. Varieties of Spanish. Pedagogical dialectology.

\section{Introducción}

Hoy en día, el español se ha convertido en una lengua global, tanto a nivel oral como escrito no solo por la cantidad de hispanohablantes que existen en los cinco continentes, sino también por su uso a nivel digital (internet, redes sociales), educativo (número de estudiantes, investigación) e incluso cultural (música, literatura, cine, etc.).

Según el último informe anual del Instituto Cervantes, se puede constatar que:

En 2018, más de 480 millones de personas tienen el español como lengua materna. A su vez, el grupo de usuarios potenciales de español en el mundo (cifra que aglutina al Grupo de Dominio Nativo, el Grupo de Competencia Limitada y el Grupo de Aprendices de Lengua Extranjera) supera los 577 millones. (INSTITUTO CERVANTES, 2018, p. 5).

Sin embargo, si continuamos observando el informe anual del Instituto Cervantes, estas cifras prevén un aumento demográfico espectacular, no solamente en los países hispanohablantes, sino en aquellos que no tienen el español como lengua oficial, principalmente en los Estados Unidos de América:

En 2060, Estados Unidos será el segundo país hispanohablante del mundo, después de México. Las estimaciones realizadas por la 
Oficina del Censo de los Estados Unidos hablan de que los hispanos serán 119 millones en 2060. Eso supondrá que el 28,6\% de la población estadounidense, casi uno de cada tres residentes en Estados Unidos, será hispano (Instituto Cervantes, 2018, p. 13).

Todo ello nos hace replantearnos la situación actual del español de los Estados Unidos que, en la actualidad, según las cifras del Instituto Cervantes (2018, p. 8), cuenta con un número de 42.125.793 millones de hablantes en el grupo de dominio nativo, y 16.075.082 millones de hablantes con una competencia limitada.

A estos datos hay que añadirle que el español cuenta con un estatus de oficialidad en territorios no incorporados como Puerto Rico y en algunos estados goza de cierto estatus a nivel administrativo, principalmente en el estado de Nuevo México. Además, la lengua española en EE. UU. dispone de una academia de la lengua propia (ANLE: Academia Norteamericana de la Lengua Española), la cual tiene como misión elaborar e implementar las reglas normativas del español en dicho país.

No obstante, debido a la coexistencia del español en un país donde el inglés es la lengua oficial y principal, la gran mayoría de los hablantes de español tienden a ser bilingües o tener, en cierto modo, conocimiento de las dos lenguas. Junto a ello, uno de los principales fenómenos que derivan de esa convivencia entre el inglés y el español es el denominado Spanglish.

Por tanto, definir el Spanglish es una tarea que durante varias décadas e incluso en la actualidad consta de mucha controversia entre diferentes lingüistas, por lo que utilizaremos la definición que ofrece el $D R A E$, en su versión en línea, como una primera aproximación sobre la materia:

\section{Espanglish $^{2}$}

Fusión de Español y el ingl. English 'inglés'.

\section{1. m. Modalidad del habla de algunos grupos hispanos de los Estados}

Unidos en la que se mezclan elementos léxicos y gramaticales del español y del inglés.

Como resultado, el presente artículo está centrado en la enseñanza del español como lengua extranjera, donde se ofrecerá una unidad didáctica, para ser realizada en el

${ }^{2}$ Lema encontrado en la versión electrónica del DRAE: https://dle.rae.es/?id=GTwoTLM, accedido el $16 / 05 / 2019$ 
aula, la cual muestre a nuestros alumnos esta variedad popular del español estadounidense.

Para ello, este trabajo está dividido en varias secciones: en la primera pretendemos mostrar una breve introducción sobre el Spanglish para poder situar al lector de este trabajo, en la segunda parte encontraremos los objetivos y las hipótesis principales que seguiremos a lo largo de la investigación. Seguidamente se detallará el marco teórico en el que se ha basado este trabajo y poder así justificar nuestra investigación. En el punto cuarto mostraremos la metodología utilizada en el aula para así, en el punto quinto y sexto, poder mostrar los resultados y conclusiones finales obtenidas.

\section{Objetivos e hipótesis}

Uno de los principales objetivos de este trabajo es transmitir en nuestra clase de E/LE esa visión global panhispánica y desmitificar que el español europeo debería ser el que aparezca, únicamente, en el aula.

Como bien describe Giménez-Folqués (2015, p. 150): [anteriormente] se consideraba al español peninsular como el que guardaba el prestigio de la lengua, pero sin duda, ante el numero de hablantes que tiene América, además del crecimiento por la cantidad de hispanohablantes en Estados Unidos, esa situación tenía que cambiar".

Partimos del punto de partida en el que en una clase de E/LE, los alumnos no solamente deben conocer aspectos gramaticales y léxicos puramente del lugar donde estudian o de la procedencia del profesor. Es necesario que los estudiantes sepan diferenciar y entender los fenómenos lingüísticos de las diferentes y principales variedades del español, por lo que:

\footnotetext{
Es mejor transmitir a nuestros alumnos en clase de español, toda esa complejidad [...] para que se acerquen a las principales diferencias, pero siempre que sea posible realizando una enseñanza lo más veraz y aproximada posible a la realidad: "lo que importa verdaderamente es conocer los rasgos que singularizan a las hablas hispanoamericanas $(\mathrm{y}$, paralelamente, los que diferencian a las variedades del español europeo), sin perder de vista lo que tienen en común, su unidad esencial. Aleza (2010: 24). (GIMÉNEZ-FOLQUÉS, 2015, p. 152).
}

Es importante añadir que el estudiante obtendrá un beneficio lingüístico, al mismo tiempo que también cultural, puesto que "llegará, sabiendo, al menos, que llega a un contexto lingüístico que, aunque sigue siendo español, pertenece a una variante 
lingüística distinta" (GIMÉNEZ-FOLQUÉS, 2015, p. 153). Además, si dispone de ese conocimiento o rasgos principales en otra variedad, "conocerá algunos esquemas básicos que le habremos enseñado $\mathrm{y}$, lo mas importante, estará predispuesto a encontrarse con rasgos lingüísticos distintos de la variante que ha estudiado" (GIMÉNEZ-FOLQUÉS, 2015, p. 153).

Con todo ello, nuestro objetivo es poder acercar al estudiante de español a otras variedades de español, y al mismo tiempo, debido al crecimiento del español en los EE. UU., hacerle ver que existe una variedad allí y que dentro de esa variedad existe una modalidad lingüística originada por las dos lenguas en contacto. Infelizmente, en la actualidad pocos libros o materiales didácticos se centran en explicar brevemente la variedad estadounidense, quedando desapercibida para el estudiante, que incluso desconoce que allí se habla español y existe una modalidad popular (Spanglish).

Por otro lado, nuestra hipótesis inicial es que, tras mostrar al estudiante de español como lengua extranjera el ámbito de las lenguas en contacto, se creará un interés intercultural que ayudará, posteriormente, a poder entender y analizar los fenómenos y recursos lingüísticos del español estadounidense y el Spanglish. Para ello, primeramente, se explicará en qué consisten dicha variedad y modalidad y después, entregarle las herramientas para no solo saber qué es, sino poder analizar por sí mismo dicha variedad, comprender los aspectos morfosintácticos y léxicos y crear así una consciencia lingüística y crítica al mismo tiempo.

\section{Marco teórico}

Es esencial que el estudiante conozca la realidad del español en Norteamérica, para ello será necesario describir la situación del español en ese territorio, datos sobre el origen, la historia, zonas dialectales y características lingüísticas propias. Como resultado, para esta primera aproximación se hará uso de la Enciclopedia del Español en los Estados Unidos (2009) como marco principal de referencia.

Posteriormente, una vez que los estudiantes dispongan de un conocimiento básico, les explicaremos que, además de tener una variedad propia, en el español estadounidense existe una modalidad híbrida popular (Spanglish) y hablaremos un poco de su ambigua definición. Debido a la problemática actual, la cual continúa en un total desacuerdo, mostraremos las tres principales definiciones del Spanglish. Por un lado, la del profesor mexicano Ilan Stavans, quien afirma que el Spanglish debería ser una lengua propia y no 
una modalidad del español o del inglés: (2000a; 2000b; 2004). Por otro lado, y contrario a la visión de Ilan Stavans, destacan lingüistas como J. Lipski (2003; 2004; 2008) o R. Otheguy (1993; 2009), quienes consideran al Spanglish como una modalidad de habla popular dentro de la variedad estadounidense del español. Finalmente, existe una tercera esfera en este ámbito de la definición y sería aquella que se centra en definir al Spanglish, no como parte del español o del inglés, sino en sus rasgos más puramente lingüísticos, como son algunos de los estudios de S. Poplack (1980), S. Betti (2008; 2011; 2013) y R. Enghels, L. Van Bellenghem y A. Vande Casteele (2019).

Asimismo, tras conocer e intentar crear las primeras intuiciones propias sobre qué es el Spanglish, nos detendremos a mostrar los recursos y procesos lingüísticos que lo forman. Para ello, mostraremos la clasificación realizada por C. Tugues (2019) basada principalmente en las obras de Fernández-Ulloa (2004), Lipski (2003) y la Enciclopedia del Español en los Estados Unidos (2009), donde encontraremos algunos recursos léxicos y morfosintácticos (préstamos, creaciones híbridas, calcos, extensiones semánticas), así como cambios de código.

Finalmente, como observaremos en la metodología, en el aula se expondrán algunas muestras reales de Spanglish en el ámbito literario. En nuestro caso, hicimos una selección de obras literarias tales como la obra poética de Tato Laviera (2014), quien fue un poeta puertorriqueño del movimiento Nuyorrican en la ciudad de Nueva York o Killer Crónicas: Bilingual Memories de Susana Chávez-Silverman (2004), escritora californiana especializada en literatura chicana. Con ello, los estudiantes tendrán que aplicar ese conocimiento mostrado en el aula de E/LE e intentar saber qué recurso lingüístico han encontrado e intentar clasificarlo.

\section{Metodología y fichas lexicográficas}

Antes de comenzar, se debe remarcar que estas actividades están deseablemente orientadas para estudiantes de E/LE en un ámbito más universitario, principalmente para aquellos alumnos que estudien un programa de grado o máster en lingüística, español o lenguas modernas y tengan materias de español como lengua extranjera o variedades dialectales del español. La justificación es que los estudiantes dispondrán de conocimientos sobre esta variedad para después aplicarlos en diferentes contextos (léxicos, morfosintácticos, etc.). 
Comenzada la clase, advertiremos a nuestros alumnos que en este día se tratará una variedad diferente. Cabe resaltar que la primera parte de la clase, orientada a tener unas dos horas, debería ser un poco más teórica y la segunda parte más práctica.

Desde nuestra propia experiencia dentro del aula, fue interesante hacer un sondeo general, preguntándoles en qué países se habla español y observar si conocían la existencia del español en los Estados Unidos. Tras ello, se les podría preguntar: “¿español en Estados Unidos o español estadounidense? Esta pregunta les hará reflexionar sobre la existencia del español allí y si es algo actual o, si por el contrario, dispone de cierta historia. Después, se pueden exponer algunos datos generales sobre la historia del español en los Estados Unidos, el número de hablantes, los estados donde más influencia tiene e incluso las variedades que influyen al español estadounidense (mexicana, puertorriqueña, centroamericana y cubana) mediante la figura que aparece en la Enciclopedia del español en los Estados Unidos:

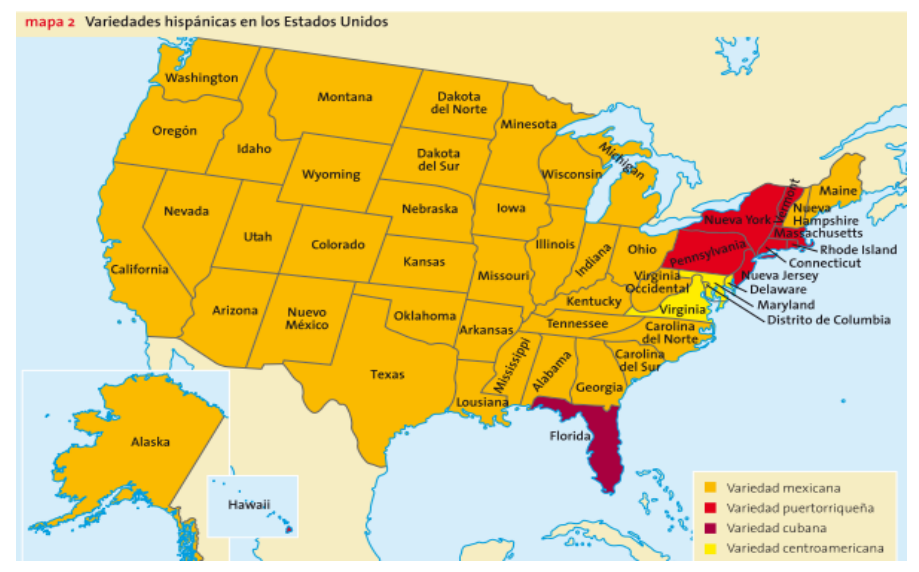

Imagen 1 - Variedades americanas en Estados Unidos ${ }^{3}$

Posteriormente, se mostrarían algunas características lingüísticas generales y propias del español estadounidense, tales como la ampliación de procesos sintácticos ya existentes, la omisión o cambio de preposiciones y algunos calcos semánticos. Finalmente, una vez que los alumnos ya dispongan de un conocimiento básico sobre el español en los Estados Unidos, se pasará a preguntarles si saben diferencian entre los siguientes dos conceptos: español culto en los Estados Unidos y español popular (Spanglish).

Esta pregunta nos servirá para distinguir y esclarecer los dos conceptos y pasar a la segunda parte más teórica, centrada en este caso en el Spanglish. Podría ser de interés común, antes de entrar en los fenómenos estrictamente más lingüísticos, mostrar algunos

${ }^{3}$ Fuente: Enciclopedia del español en los Estados Unidos (2009). 
ejemplos léxicos como iniciación (anuncios, muestras léxicas en obras literarias, comentarios de Facebook, etc.), así como recursos digitales para observar la entonación, la sonoridad y el acento (canciones o vídeos):

Tabla 1 - Ejemplos léxicos del Spanglish y su traducción al inglés y español

\begin{tabular}{|c|c|c|}
\hline Inglés & Spanglish & Español \\
\hline Ceiling / Roof & Rufa & Tejado \\
\hline Market & Marketa & Mercado \\
\hline To mop & Mopear / Mopiar & Pasar la fregona \\
\hline
\end{tabular}

Con todo ello, una vez que los alumnos son capaces de identificar el fenómeno del Spanglish, sería interesante exponer la problemática actual respecto a su definición. Para ello se deberían mostrar las tres teorías e hipótesis sobre qué es Spanglish (la teoría del Spanglish como lengua, la segunda teoría del Spanglish como dialecto o variedad del español y finalmente aquella que afirma que el Spanglish es un elemento sociocultural). Posteriormente, podríamos preguntar a nuestros alumnos sobre sus propias creencias en lo que atañe al Spanglish y que argumenten, según las tres teorías, cual sería su visión y el porqué.

En el siguiente paso de la unidad didáctica tendría lugar la explicación, dentro del aula, de los principales procesos y fenómenos lingüísticos que aparecen bajo la etiqueta de Spanglish. En primer lugar, el profesor les mostraría la lista de C. Tugues (2019, p. 36-37) y explicaría los diferentes procesos y recursos, para que finalmente la apliquen en la actividad posterior. Algunos de los recursos que aparecen en dicha lista son:

\section{Préstamos:}

1.1 Préstamos puros: tiene el pelo straight.

1.2 Creaciones híbridas: calendador, troka.

a) Preposiciones: conocí mi esposa. 
b) Verbos y Gerundios: lunchear, spikear, empecé a mirando la TV.

1.3 Calcos: maquina de responder, fuerza policía.

a) gramaticales:

Pérdida o desaparición de recursos y mecanismos sintácticos.

Ampliación en el uso de procesos sintácticos ya existentes.

1.4 Extensión Semántica (Fraseología): ¿Qué tiempo es?, ¿Qué es tu nombre?

\section{Cambio de código:}

2.1 Cambio intersentencial: decir una frase en una lengua y la siguiente en otra. No vas a decir nada... You are not gonna say anything.

2.2 Cambio intrasentencial: el cambio se realiza dentro de la misma cláusula. I told him that pa'que la trajera ligero.

Finalmente, una vez que los estudiantes disponen de los conocimientos necesarios para poder analizar linguísticamente los recursos y procesos del Spanglish, sería tarea del profesor mostrar varias muestras escritas en Spanglish, para poder, posteriormente, completar un corpus o fichas lexicográficas.

En nuestro caso, para la aplicación de la unidad didáctica en el aula de español como lengua extranjera, trajimos muestras reales de la colección poética del poeta puertorriqueño afincado en Nueva York, Jesús Tato Laviera.

Una vez seleccionada las muestras, les entregamos unas fichas lexicográficas con ejemplos, donde aparecerá la siguiente información: 
Tabla 2 - Ejemplo de ficha lexicográfica

\begin{tabular}{|c|c|}
\hline Entrada & \multicolumn{1}{|c|}{ Marketa } \\
\hline Contexto & $\begin{array}{l}\text { "Un Moreno vende wholesale marketa de } \\
\text { lipsticks por diez dólares". }\end{array}$ \\
\hline Traducción & $\begin{array}{l}\text { "Un Moreno mayorista vende barras de } \\
\text { labios en el supermercado por diez } \\
\text { dólares". }\end{array}$ \\
\hline Tipo de fenómeno & \multicolumn{1}{|c|}{ Préstamo. (en blanco) } \\
\hline Subtipo & \multicolumn{1}{|c|}{ Social Club, pág. 173. } \\
\hline Fuente & \begin{tabular}{c} 
DDA: marketa, marqueta: supermercado. \\
\hline Diccionarios
\end{tabular} \\
\hline Origen & $\begin{array}{l}\text { Existe la acepción supermarketa. } \text { Para } \\
\text { formar la palabra marketa, el hablante } \\
\text { añade la vocal 'a' al final de la palabra en } \\
\text { inglés. Ocurre lo mismo con palabras como } \\
\text { laptopa (odenador portátil) o capa (gorra). }\end{array}$ \\
\hline
\end{tabular}

Como podemos observar en las fichas lexicográficas, el alumno encontrará la entrada en su contexto original dentro del poema junto a una traducción en español. Seguidamente aparecerán dos casillas en blanco (el tipo de fenómeno y el subtipo si lo hubiere). Esas dos casillas deberían ser completadas por el alumno usando así sus conocimientos, adquiridos recientemente, sobre los procesos lingüísticos del Spanglish.

Después, aparecerá otra información relativa a las entradas para constatar la información: fuente de origen, diccionarios (si estuviese registrado), el posible origen de la palabra y finalmente las observaciones respectivas.

La finalidad de la tarea es que, tras mostrar al estudiante las diferentes muestras reales junto a la información pertinente, el estudiante sea capaz de reconocer las diferentes muestras y usar el conocimiento adquirido para poder así seleccionar y clasificar las muestras bajo los diferentes tipos de fenómenos y sus subtipos.

\section{Resultados principales}

De entre los resultados principales obtenidos, uno de los más destacables fue el claro interés y recepción de los estudiantes en relación al Spanglish. Muchos de ellos 
desconocían el mundo de las lenguas en contacto por lo que se mostraron bastante receptivos y motivados en respecto a esta modalidad lingüística y ante la variedad del español estadounidense

Otro resultado, también muy gratificante, fue que los alumnos, los cuales tienen un nivel de usuario medio de inglés y español, fueron capaces de reconocer bastantes aspectos léxicos y palabras en Spanglish, por lo que también fue mucho más fácil poder realizar las actividades prácticas. Además, aprendieron nuevos aspectos y procesos léxicos y morfosintácticos que no se suelen estudiar, por norma general, en las materias de morfología, fonología, lexicografía o sintaxis, aprendiendo así, no solamente elementos de la lengua española, sino también lingüísticos, estrictamente hablando.

Como resultado, los alumnos no encontraron una gran dificultad en clasificar las muestras dentro de las fichas lexicográficas, eligiendo y explicando el porqué de cada tipo y subtipo. Entre todas las muestras, ellos fueron capaces de encontrar y distinguir préstamos, creaciones híbridas, extensiones semánticas, préstamos puros y cambios de código. Algunos ejemplos de sus respuestas fueron los siguientes:

Tabla 3 - Bipeó

\begin{tabular}{|c|c|}
\hline Entrada & \multicolumn{1}{|c|}{ Bipeó > Bipear } \\
\hline Contexto & $\begin{array}{l}\text { "Ella me bipeó en la celular she told me } \\
\text { that deep into el yunque rain forest an } \\
\text { international gathering of coquís". }\end{array}$ \\
\hline Tipo de fenómeno & \multicolumn{1}{c|}{ Préstamo. } \\
\hline Subtipo & Creación Híbrida. \\
\hline Fuente & DDA: bipear. Enviar mensajes por un bíper. \\
\hline Diccionarios & Del inglés Beeper. \\
\hline Origen & $\begin{array}{c}\text { Se adapta la doble 'e' por su pronunciación } \\
\text { /i/ y se añade la desinencia -ear. }\end{array}$ \\
\hline Observaciones &
\end{tabular}


Tabla 4 - Craqueao

\begin{tabular}{|c|c|}
\hline Entrada & Craqueao $>$ Craquear / Craquearse \\
\hline Contexto & $\begin{array}{l}\text { "Darwin está muerto. iSeguro!, y tú } \\
\text { craqueao!". }\end{array}$ \\
\hline Tipo de fenómeno & Préstamo. \\
\hline Subtipo & Creación Híbrida. \\
\hline Fuente & Craqueao, pág. 226. \\
\hline Diccionarios & Del inglés to crack up. \\
\hline Origen & $\begin{array}{c}\text { Según el } D D A, \text { a pesar de utilizarse en } \\
\text { varios países hispanoamericanos, tiene un } \\
\text { mayor uso en Puerto Rico. }\end{array}$ \\
\hline Observaciones & $\begin{array}{r}\text { marse (roto). } \\
\hline\end{array}$ \\
\hline
\end{tabular}

Tabla 5 - Soy torno atrás

\begin{tabular}{|c|c|}
\hline Entrada & Soy torno atrás \\
\hline Contexto & $\begin{array}{c}\text { "Soy torno atrás de Rafael cortijo los } \\
\text { soneos dulces creativos de ismael rivera". }\end{array}$ \\
\hline Tipo de fenómeno & Préstamo. \\
\hline Subtipo & Eestensión semántica. \\
\hline Fuente & Degreza, pág. 288. \\
\hline Diccionarios & $\begin{array}{c}\text { Es inusual encontrar en inglés ese verbo } \\
\text { junto al verbo to be. Aparentemente, existe } \\
\text { su uso en el Spanglish, principalmente de la } \\
\text { zrigen }\end{array}$ \\
\hline Observaciones de Nueva York. \\
\hline
\end{tabular}

\section{Conclusiones}

Una de las principales conclusiones que podemos extraer de este trabajo es la justificación de llevar las distintas variedades de una lengua al aula. Pensamos que es estrictamente necesario que el profesor, además de que mostrar su variedad en el aula, debe acercar al estudiante a todas las variedades que una lengua puede ofrecer, aunque sea de una manera breve, creando esa consciencia lingüística en sus alumnos. 
En otras palabras, el alumno tiene que ser consciente de que, si viaja a un lugar, o escucha esa variedad en la televisión, debe estar predispuesto a poder comprender los elementos lingüísticos y usar las claves aprendidas en el aula para así establecer una situación comunicativa efectiva.

Asimismo, el hecho de traer al aula de E/LE o a un aula de dialectología hispánica en un contexto universitario una lengua en contacto, como podría ser el Spanglish o el Portuñol, hace que el estudiante conozca una dimensión intercultural nueva, donde las lenguas no son algo ya establecido, sino más bien en cambio constante, eliminando cualquier tipo de preconcepto lingüístico. Además, haciendo más visible el contacto lingüístico en las aulas de lenguas, se conseguirá crear más material, unidades didácticas y actividades, eliminando así el tabú o la creencia de que las lenguas en contacto tienen menos prestigio que otras variedades o modalidades de habla.

Al mismo tiempo, en nuestro caso, hemos conseguido que nuestros alumnos se interesasen por un campo en la lingüística, nuevo para la gran mayoría de ellos, pudiendo así crear una nueva perspectiva en sus trabajos de investigación, al mismo tiempo que aprendieron otra variedad del español.

\section{REFERENCIAS}

ALEZA. M.; ENGUITA, J. La Lengua Española en América: normas y usos actuales. Valencia: Universitat de València, 2010. Disponible en:

http://www.uv.es/aleza/esp.am.pdf. Accedido el: 03 abr. 2019.

BETTI, S. El Spanglish ¿medio eficaz de comunicación? Bologna: Pitagora Editrica, 2008.

BETTI, S. El Spanglish en los Estados Unidos: ¿estrategia expresiva legítima? en Lenguas Modernas 37 (Primer Semestre 2011), p. 33-53, 2011. Disponible en: http://www.lenguasmodernas.uchile.cl/index.php/LM/article/download/30687/32443. Accedido el: 02 abr. 2019.

BETTI, S. La ilusión de una lengua: el Spanglish entre realidad y utopía. En Domnite Dumitrescu y Gerardo Piña-Rosales (eds.) New York: Academia Norteamericana de la Lengua Española (ANLE), p. 189-216, 2013.

CHÁZVEZ-SILVERMAN, S. Killer Crónicas. Wisconsin: University of Wisconsin Press, 2004. 
ENGHELS, R.; VAN BELLEGUEM. L.; VANDE CASTEELE, A. ¿Existe una gramática del spanglish? Estudio de caso de la posición del adjetivo. En Killer Crónicas (2004) en Silvia Betti y Renata Enghels, El inglés y el español en contacto en los Estados Unidos. Reflexiones acerca de los retos, dilemas y complejidad de la situación lingüística estadounidense. Roma: Aracne Editice, 2019.

FERNÁNDEZ-ULLOA, T. Espanglish y cambio de código en alumnos de high school de Shafter, California. Bakersfield: BilingLatAm. Symposium Proceedings, ESSARP, p. 82-94, 2004. Disponible en:

http://www.csub.edu/ tfernandez_ulloa/CAMBIO\%20DE\%20CODIGO.pdf. Accedido el: 14 fev. 2019.

GIMÉNEZ FOLQUÉS, D. ¿Es conveniente enseñar las variantes del español en clase de E/LE? En Felisa Bermejo, Americanismos en lexicografía y en español/LE. Una mirada abierta. RiCOGNIZIONI. Rivista di lingue, letterature e culture moderne, 3 , p.149-155, 2015(II).

GIMÉNEZ FOLQUES, D. La enseñanza de la gramática panhispánica en clases de español como lengua extranjera (E/LE). Cauce: Revista Internacional de Filología, Comunicación y sus Didácticas, número 40, p. 88-95. Sevilla, 2017.

INSTITUTO CERVANTES. Informe anual 2018: el español, una lengua viva. Instituto Cervantes, 2018. Disponible en:

https://cvc.cervantes.es/lengua/espanol_lengua_viva/pdf/espanol_lengua_viva_2018.pdf

LAVIERA, T. Bendición: The Complete Poetry of Tato Laviera. Texas: Arte Público, 2014.

LIPSKI, J. La lengua española en los Estados Unidos: avanza a la vez que retrocede, Revista Española de Lingüística, v. 22, n. 2, p. 231-260, 2003. Disponible en: http://www.personal.psu.edu/faculty/j/m/jml34/SEL.PDF . Accedido el: 18 mar. 2019

LIPSKI, J. El español de América: los contactos bilingüe, en Rafael Cano (coord.) Historia de la lengua española. Barcelona: Ariel, p. 1117-1138, 2004.

LIPSKI, J. Varieties of Spanish in the United States. Washington, D.C.: Georgetown University Press, 2008.

LÓPEZ, H. Enciclopedia del Español en los Estados Unidos. Alcalá de Henares: Instituto Cervantes / Santillana, 2009.

OTHEGUY, R. Convergent conceptualizations as predictors of degrees of contact in U.S. Spanish. En Ana Roca y John Lipski, Spanish in the United States: Linguistic Contact and Diversity. Nueva York: Mouton de Gruyter. p. 135-154, 1993.

OTHEGUY, R. El llamado Espanglish. En López Morales, Humberto, Enciclopedia del Español en los Estados Unidos. Alcalá de Henares: Instituto Cervantes / Santillana, p. 222-243, 2009. 
REAL ACADEMIA ESPAÑOLA. Diccionario de la Lengua Española. Madrid: Espasa, 2001.

STAVANS, I. Spanglish: tickling the tongue, World Literature Today, v. 74, n. 3, p. 555-9, 2000. Disponible en:

http://isites.harvard.edu/fs/docs/icb.topic653576.files/Spanglish\%20Tickling\%20the \%20Tongue.pdf. Accedido el: 31 jan. 2019.

STAVANS, I. The Gravitas of Spanglish, The Chronicle Review 13 Oct. 2000b. Disponible en: http://chronicle.com/free/v47/i07/07b00701.htm. Accedido el: 30 jan. 2019.

STAVANS, I. Spanglish: The Making of a New American Language, Nueva York, HarperCollins, 2004.

TUGUES, C. Los Recursos Lingüísticos del Spanglish en los Estados Unidos de América: un análisis de la colección poética de Tato Laviera. Valencia: Edelex Editorial Colección Estudios, 2019.

POPLACK, S. Sometimes I'll start a sentence in English y termino en español. Linguistics 18, p. 581-618.

\section{Cómo citar este artículo}

RODRIGUEZ, C. T. Los aspectos morfosintácticos y léxicos del Spanglish en la clase de E/LE en un contexto universitario. Rev. EntreLínguas, Araraquara, v. 5, n. esp. 1, p. 282-296, jul., 2019. E-ISSN: 2447-3529. DOI: 10.29051/el.v5iesp.1.12986

Submetido em: 01/06/2019

Aprovado em: 30/06/2019

Publicado em: 01/10/2019 